\title{
Practical Laboratory Tools for Monitoring of BCR-ABL1 Transcripts and Tyrosine Kinase (TK) Domain Mutations in Chronic Myeloid Leukemia Patients Undergoing TK Inhibitor Therapy: A Single-Center Experience in Thailand
}

\author{
Nittaya Limsuwanachot ${ }^{1}$, Adcharee Kongruang ${ }^{1}$, Budsaba Rerkamnuaychoke ${ }^{1}$, \\ Roongrudee Singdong ${ }^{2}$, Pimjai Niparuck ${ }^{3}$, Saengsuree Jootar ${ }^{3}$, Teerapong \\ Siriboonpiputtana ${ }^{1 *}$
}

\begin{abstract}
Objective: The genetic hallmark of CML is known as the appearance of $\mathrm{t}(9 ; 22)(\mathrm{q} 34.1 ; \mathrm{q} 11.2)$ (BCR-ABL1) which is present in more than $95 \%$ of cases. Here, we demonstrated practical laboratory tools for monitoring of BCR-ABL1 transcripts in chronic myeloid leukemia patients undergoing TK inhibitor therapy. Methods: Real time quantitative PCR and direct sequencing were performed for monitoring of BCR-ABL1 transcripts in 245 treated CML. Results: At month 3 after first time point of monitoring, we found that 89\% (218/245), 2\% (5/245), and 9\% (22/245) of patients are determined as optimal, warning, and failure response, respectively. The responses to TKI were slightly decreased at months 6 as following 73\% optimal (180/245), 18\% warning (43/245), and 9\% failure response (22/245). Additionally, responses to TKI were gradually decreased at month 12 after first time point of monitoring as following $65 \%$ optimal (160/245), $13 \%$ warning (31/245), and $22 \%$ failure (54/245). We could detect $20 \%(49 / 245)$ of patients positive for BCR-ABL1 TKD mutations. Interestingly, one third (17 of 49) of TKD mutated cases were positive for compound/polyclonal mutation patterns. While major molecular response were observed in the majority of patients without TKD mutation, resistant to TKI were detected in patients with T315I mutation ( $\mathrm{n}=9 ; \%$ mean IS $=8.1510, \%$ median IS = 9.7000), compound/polyclonal mutations with T315I ( $\mathrm{n}=9 ; \%$ mean IS $=13.0779, \%$ median IS $=5.404)$, and other TKD mutations ( $\mathrm{n}=14 ; \%$ mean IS $=8.1416, \%$ median IS $=1.060)$, respectively. Conlusion: These practical laboratory techniques provided a more comprehensive understanding of CML progression during drug therapy and could be of benefit in earlier prognosis.
\end{abstract}

Keywords: Chronic myeloid leukemia- BCR-ABL1 mRNA- BCR-ABL1 TKD mutations

Asian Pac J Cancer Prev, 21 (7), 2003-2012

\section{Introduction}

Chronic myeloid leukaemia is a clonal haematopoietic stem cells disorder characterized by the proliferation of malignant myeloid cells at all stages of differentiation. The genetic hallmark of CML is known as the appearance of the Philadelphia chromosome (Ph chromosome); $\mathrm{t}(9 ; 22)$ (q34.1; 111.2 ) which is present in more than $90 \%$ of cases. The $\mathrm{Ph}$ chromosome is associated with the production of BCR-ABL1 chimeric protein with high ABL tyrosine kinase activity and recognized as a pathogenesis of the disease (Jabbour et al., 2008; Thompson et al., 2015). Patients with CML frequently have three phases of disease progression including the initial chronic phase, the transitional accelerated phase, and the progressive blast crisis phase (Calabretta and Perrotti, 2004; Tantiworawit et al., 2016). Since the discovery of imatinib (IM) (Baccarani et al., 2013; Buchdunger et al., 2001; Druker and Lydon, 2000), the drug has been shown to be the highly effective therapy in $\mathrm{Ph}$ positive CML. IM specifically blocks the ATP-binding site of BCR-ABL1 protein in leukemic cells resulting in inhibiting downstream signaling pathways. The first application of IM was to cure CML patients who were resistant to interferon-alpha (IFN $\alpha)$ (Goldman and Melo, 2003; Savage and Antman, 2002). The drug can induce a rapid haematological response and a major

${ }^{1}$ Human Genetic Laboratory, Department of Pathology, Faculty of Medicine Ramathibodi Hospital, Mahidol University, Bangkok, Thailand. 'Doctoral Program in Clinical Pathology, Department of Pathology, Faculty of Medicine Ramathibodi Hospital, Mahidol University, Bangkok, Thailand. ${ }^{3}$ Department of Medicine, Faculty of Medicine Ramathibodi Hospital, Mahidol University, Bangkok, Thailand.*For Correspondence: teerapong.sir@mahidol.ac.th 
cytogenetic response in a chronic phase CML patients (Druker et al., 2001; Lim et al., 2017). In addition, IM could rise the overall survival and the event-free survival in the accelerated phase CML (Talpaz et al., 2002). Moreover, adverse effects of IM are generally mild when compared with interferon-alpha. IM could dramatically improve the overall survival of CML patient from about $20-80 \%$ to about $80-90 \%$. Presently, treated CML patients are expected to live longer as well as have very well quality of life. Therefore, monitoring of the persistence specific surrogate disease biomarkers in CML (Ph chromosome, BCR-ABL1 fusion gene, and BCR-ABL1 mRNA) is very important during a treatment to prevent disease relapse as well as to establish the end-point of therapy (Jabbour and Kantarjian, 2014). Recently, the European LeukemiaNet recommendations for the management of chronic myeloid leukemia suggested that bone marrow studies including cytogenetic analysis should be performed at 3,6, and 12 months after starting therapy (Baccarani et al., 2013). Additionally, the improvement of clinical outcomes in treated CML patient are strongly associated with the deep and rapid molecular response. Hence, highly specific and sensitive techniques such as RT-PCR and real-time quantitative PCR (RQ-PCR) are important for routine monitoring of minimal residual disease (MRD) in treated patients (Breccia et al., 2016). Although IM has been proved for the effective treatment of CML, approximately to $20-30 \%$ of treated patients become IM resistance (Yang and Fu, 2015). Those patients become predominantly increasing in the BCR-ABL1 level. The resistance to IM treatment in CML patients could be categorized into two main mechanisms. Firstly, a BCR-ABL1 dependent mechanism which is characterized by aberrations on the BCR-ABL1 fusion gene, including $B C R-A B L 1$ kinase domain mutations (the most common cause of IM resistance), BCR-ABL1 amplification, and clonal evolution. Secondly, a BCR-ABL1 independent mechanism, which includes the impairment in function of specific IM transporters (influx and efflux transporters), drug concentration, the dysregulation of alternative signaling pathways, and epigenetics (An et al., 2010; Bixby and Talpaz, 2009; Chhikara et al., 2017; Cooper et al., 2009; Elias et al., 2013; Patel et al., 2017; Shah et al., 2016; Tauchi and Ohyashiki, 2004). At present, it is clear that the mutation in $A B L 1$ tyrosine kinase domain (TKD) is the major cause of IM resistance (Kaleem et al., 2015; Vacarean-Trandafir et al., 2019). Common TKD mutations are including the highly resistant mutations $(Y 253 F / H, E 255 K / V, T 315 I$, or $H 396 P / R)$ which are recommended to alternative treatment options, the mildly resistant mutations ( $M 244 \mathrm{~V}, \mathrm{M351T}$, and $\mathrm{F359}$ ) which permit the improvement of clinical outcomes by increasing the IM dose to 600 or $800 \mathrm{mg}$ /day (Hehlmann et al., 2007). Thus, molecular methods that can be able to early detect $B C R-A B L 1$ kinase domain mutations are very important for monitoring of CML patients after treatment with tyrosine kinase inhibitors (TKIs). In this report, we described our routine RQ-PCR for the measurement of BCR-ABL1 mRNA and PCR sequencing technique for the detection of $B C R-A B L 1 T K D$ mutations in CML patients during TKIs treatments. Finally, we reported the frequency of $B C R-A B L 1 T K D$ mutations and the impact of clinical important TKD mutations on molecular response to TKIs treatment in Thai patients with CML.

\section{Materials and Methods}

\section{Patients and samples}

This study was a retrospective analysis on a total of 245 CML patients during treatment at the Faculty of Medicine, Ramathibodi Hospital, Mahidol University, Bangkok, Thailand during January 2011 to December 2016. Cytogenetic study, $R Q-P C R$, and $B C R-A B L 1$ kinase domain mutations screening were performed by the request from hematologists. The management of CML were based on the recommendations of European LeukemiaNet (Baccarani et al., 2009; Baccarani et al., 2006). Bone marrow and peripheral blood specimens were collected from patients at the time of diagnosis as well as during treatment. Standard karyotyping was mainly performed using bone marrow samples. The quantification of BCR-ABL1 mRNA levels were conducted by following the EAC protocol (Gabert et al., 2003). BCR-ABL1 kinase domain mutations analysis by PCR sequencing was performed on cDNA generated from RNA isolated from both peripheral blood and bone marrow samples. This work was approved by the ethic committee on human right related to research involving human subjects, Faculty of Medicine Ramathibodi Hospital, Mahidol University, Thailand and followed the principles of the Declaration of Helsinki (ID; MURA2020/174).

\section{Real-time quantitative PCR}

TaqMan-based real-time quantitative PCR (RQ-PCR) was performed according to a Europe Against Cancer Program (EAC) protocol to measure the BCR-ABL1 mRNA level during treatment (Gabert et al., 2003). Briefly, total RNA was isolated from peripheral blood/ bone marrow samples of each patient by using TRIzol@ Reagent (Thermo Fisher Scientific, USA) according to the manufacturer's instructions and subsequently measured the concentration by using the Nanodrop 2000 spectrophotometer (Thermo Scientific, USA). cDNA was generated from a total of $1 \mu \mathrm{g}$ RNA using SuperScript ${ }^{\mathrm{TM}}$ VILO $^{\mathrm{TM}}$ cDNA Synthesis Kit (Thermo Fisher Scientific, USA) with random oligonucleotide primers. Real time quantitative PCR was performed on ABI 7500 Fast Real-Time PCR System (Thermo Fisher Scientific, USA). ABL1 was used as a house-keeping gene. BCR-ABL1/ABL1 copy ratio and \% IS (international scale) of individual patient during treatment (every 3 months) were reported. The major molecular response (MMR) was defined as $\geq 3-\log$ reduction ( $\%$ IS $\leq 0.01$ ) in BCR-ABL1/ABL1 ratio. The laboratory has achieved ISO15189 and 15190 accreditations and participated in the College of American Pathology (CAP) External Quality Assurance (EQA)/Proficiency Testing program.

\section{The definition of response to TKIs}

The definition of response to any TKIs was followed the ENL 2013 recommendation guideline for the management of CML (Baccarani et al., 2013). The response to TKIs of 
individual patient could be assessed by both cytogenetic and molecular (RQ-PCR) techniques. The specific cutoff values at diverse time points of monitoring were clearly written in the guideline. The optimal response was associated with the achievement of finest long-term outcomes. Therefore, in this case, it was not necessary to change the treatment. In contrast, failure response meant that patient should change the treatment strategies to avoid the risk of disease progression and death. The middle between optimal and failure responses is called warning (previous known as sub-optimal response). In this group, patients required more frequent monitoring to allow appropriate changes for new treatments in a case of failure response. The guideline was able to be used for the definition of response to TKIs in all phases of CML (chronic, accelerated, and blast phases) and could be applied for the definition of response in second-line TKIs treatments.

\section{$B C R-A B L 1$ kinase domain mutation analysis}

PCR sequencing analysis for the detection of $B C R-A B L 1$ kinase domain mutations was adapted from the previous published protocol by Branford and Hughes, (2006) and Gabert et al., (2003). Briefly, semi-nested RT-PCR was performed to initially amplify BCR-ABL1 fusion transcripts (ENF501 and ABLR1) and subsequently amplify the ABL1 kinase domain in a second round of PCR (ABLF1 and ABLR1). The PCR primers used in both semi-nested PCR and sequencing reaction were list in Table 1. The optimal PCR condition for both first and second rounds of semi-nested PCR was following the initial PCR with $95^{\circ} \mathrm{C}$ for 10 minutes, 40 cycles of $94^{\circ} \mathrm{C}$ for 1 minute, $68^{\circ} \mathrm{C}$ for 1 minute, $72^{\circ} \mathrm{C}$ for 2 minutes, and final extension at $72^{\circ} \mathrm{C}$ for 10 minutes. $5 \mu \mathrm{l}$ of the first round PCR product was used as a template in the second round PCR. PCR product sizes of 1,464 or 1,539 base pairs for the first round PCR and 863 base pairs for the second round PCR were determined by $2 \%$ agarose gel electrophoresis. PCR product ( $863 \mathrm{bp}$ ) was subsequently purified by using ExoSAP-ITTM PCR Product Cleanup Reagent (Thermo Fisher Scientific, USA) prior to cycle sequencing process. Sequencing reaction was performed by using BigDye ${ }^{\circledR}$ Terminator v1.1 Cycle Sequencing Kit (Thermo Fisher Scientific, USA) and $1.6 \mathrm{pmol}$ of each sequencing primer (ABLF1 and ABLR1). The amplified condition was following $96^{\circ} \mathrm{C}$ for 1 minute, 25 cycles of $96^{\circ} \mathrm{C}$ for 10 second, $50^{\circ} \mathrm{C}$ for 5 seconds, $60^{\circ} \mathrm{C}$ for 4 minutes. Sequencing product was purified using DyeEx 2.0 Spin Kit (QIAGEN, Germany) and subsequently sequenced with ABI 3130 Genetic Analyzer (Applied Biosystems, USA) according to the instruction protocol. Finally, sequence was compared to the wild-type ABL sequence (GenBank accession number X16416.1).

\section{Statistical analysis}

Statistical analysis was performed using SPSS version 24.0 software (SPSS Inc., Chicago, IL, USA). Mean and median percent IS values of patients at 12 months of monitoring were analysed using independent T-test and Mann-Whitney test respectively. Statistical significance is accepted at $\mathrm{p}$-values $<0.05$.

\section{Results}

The overall molecular response of 245 CML patients to TKI treatment

We performed both cytogenetic analysis and real-time quantitative PCR on sequential samples collected from different time points of individual patients during treatment. The definition of response to tyrosine kinase inhibitors as a first-line treatment was based on the European LeukemiaNet recommendations for the management of chronic myeloid leukemia; 2013 (Baccarani et al., 2013). At month 3 of monitoring, we found that $89 \%(218 / 245), 2 \%(5 / 245)$, and $9 \%(22 / 245)$ of patients were determined as optimal, warning, and failure response, respectively. The overall responses to TKI treatment were slightly decreased at the month 6 of disease monitoring as following $73 \%$ of patients with optimal response $(180 / 245), 18 \%$ of patients with warning (43/245), and $9 \%$ of patients with failure response $(22 / 245)$. Additionally, we observed that the overall

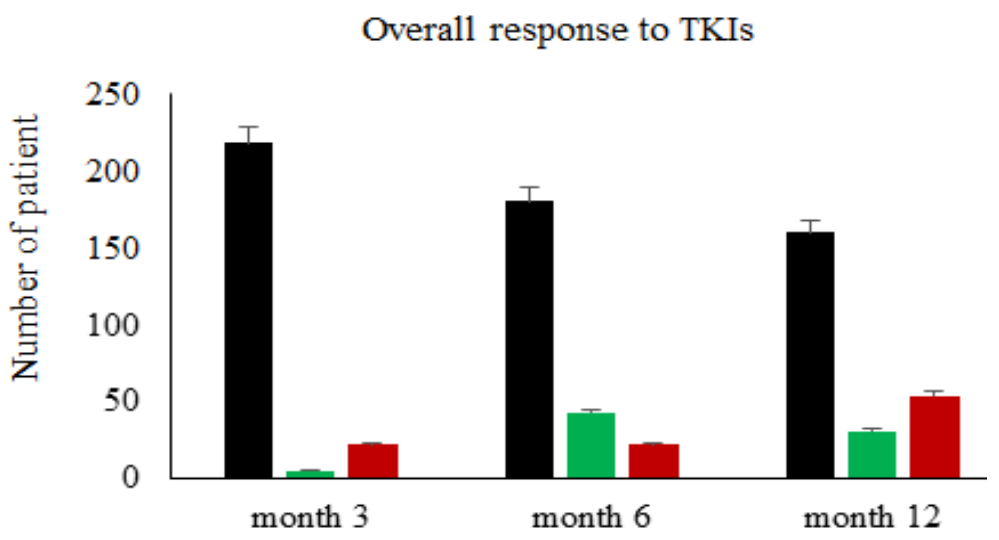

Figure 1. Clinical Response Categories of Chronic Myeloid Leukemia Patients $(n=245)$ to a 12-Month Tyrosine Kinase Inhibitor (TKI) Treatment at Ramathibodi Hospital, Mahidol University, Bangkok, Thailand. Definition of clinical response to TKI treatment was based on ENL 2013 recommendation guideline for management of CML (Baccarani et al., 2013). The colored-bar boxes indicate number of patient's response to TKIs at specific time points of monitoring. The black boxes were patients with optimal response, green boxes were warning groups, and red boxes were patients with failure response to TKIs, respectively. 
Table 1. Primers for Semi-Nested RT-PCR and Direct Sequencing of $B C R-A B L 1$ TKD

\begin{tabular}{llc}
\hline Name & Sequence $\left(5^{\prime}-3^{\prime}\right)$ & PCR process \\
\hline ENF501 & TCCGCTGACCATCAAYAAGGA & $1^{\text {st }}$ round \\
ABLR1 & TCCACTTCGTCTGAGATACTGGATT & $1^{\text {st }}$ round, 2nd and sequencing \\
ABLF1 & CGCAACAAGCCCACTGTCT & $2^{\text {nd }}$ round, and sequencing \\
\hline
\end{tabular}

responses to TKI treatment were gradually decreased at month 12 of monitoring as following $65 \%$ of patients with optimal response $(160 / 245), 13 \%$ of patients with warning $(31 / 245)$, and $22 \%$ of patients with failure response (54/245) (Figure 1). BCR-ABL1 TKD mutations analysis was subsequently confirmed in cDNA samples of patients who exhibited signs of treatment failure. Using quantitative RT-PCR, we could determine the molecular response of CML patients after TKI treatment.

Mutation spanning of BCR-ABL1 TKD in treated CML patients

$B C R-A B L 1$ TKD mutations analysis by Sanger sequencing was performed in cDNA samples of patients with TKIs treatment failure. We could detect $20 \%(49 / 245)$ of patients with positive results for $B C R-A B L 1$ TKD mutations. Interestingly, we found that one third (17 of 49) of TKD mutated cases were positive for compound/polyclonal mutation patterns. An example of electropherogram from patient with compound/ polyclonal mutation was presented in Figure 2. The most frequent point mutation of $B C R-A B L 1$ TKD was the clinical relevant, T315I which was positive in 57\% (28/49) of all mutated cases. Interestingly, compound/polyclonal mutations of $T 315 I$ with other BCR-ABL1 TKD mutations were observed in nearly half of T315I mutated cases (12 of $28,43 \%$ ). Other point mutation types observed in this study were following $20 \%$ of E255K/V (10/49; 4 single and 6 compound/polyclonal mutations), $10 \%$ of F359V/I (5/49; 2 single and 3 compound/polyclonal mutations), $8 \%$ of $\mathrm{F} 317 \mathrm{~L} / \mathrm{I}$ (4/49; 3 single and 1 compound/polyclonal mutations $), 6 \%$ of $Y 253 \mathrm{H}(3 / 49 ; 1$ single and 2 compound/ polyclonal mutations), 4\% of D276G (2/49; 1 single and 1 compound/polyclonal mutations), $4 \%$ of $\mathrm{E} 459 \mathrm{~V} / \mathrm{K}$ (2/49), 2\% of Q252H (1/49), 2\% of F311I (1/49), 2\% of A350T (1/49), 2\% of E455K (1/49), 2\% of F486S (1/49), respectively. Interestingly, $G 250 E$ mutation was restricted positive as compound/polyclonal mutation patterns. There was no single mutation of G250E mutation observed in this study (Table 2).

\section{BCR-ABL1 TKD mutations and molecular response to} TKI treatment

We further investigated the impact of $B C R-A B L 1 \mathrm{TKD}$ mutations on the overall molecular response (by means of IS scale) to TKIs at month 12 of monitoring. 142 patients without $B C R-A B L 1$ TKD mutation were analysed. We observed that the median \% IS of patients at month 12 is $0.0150(<-4 \log )$. This data suggested the majority of patients was achieved major molecular response after TKI treatment. However, there were few patients with a sign of molecular resistance at one year after treatment without $B C R-A B L 1$ TKD mutation. Because of T315I is well-recognized as a clinical significant point mutation that contributes to imatinib resistant in CML and it was predominantly observed in this study ( $57 \%$ of all mutated cases), we classified the treated patients into 4 main groups including patients without $B C R-A B L 1$ TKD mutation, T315I mutation, compound/polyclonal mutations with T315I, and other TKD mutations (such as E255K, $F 317 \mathrm{~L}, \mathrm{F359V} / \mathrm{I}$, and F486S). While major molecular response was observed in the majority of patients without $B C R-A B L 1$ TKD mutation, resistance to TKI treatments was detected in patients with $T 315 I$ mutation $(\mathrm{n}=9 ; \%$
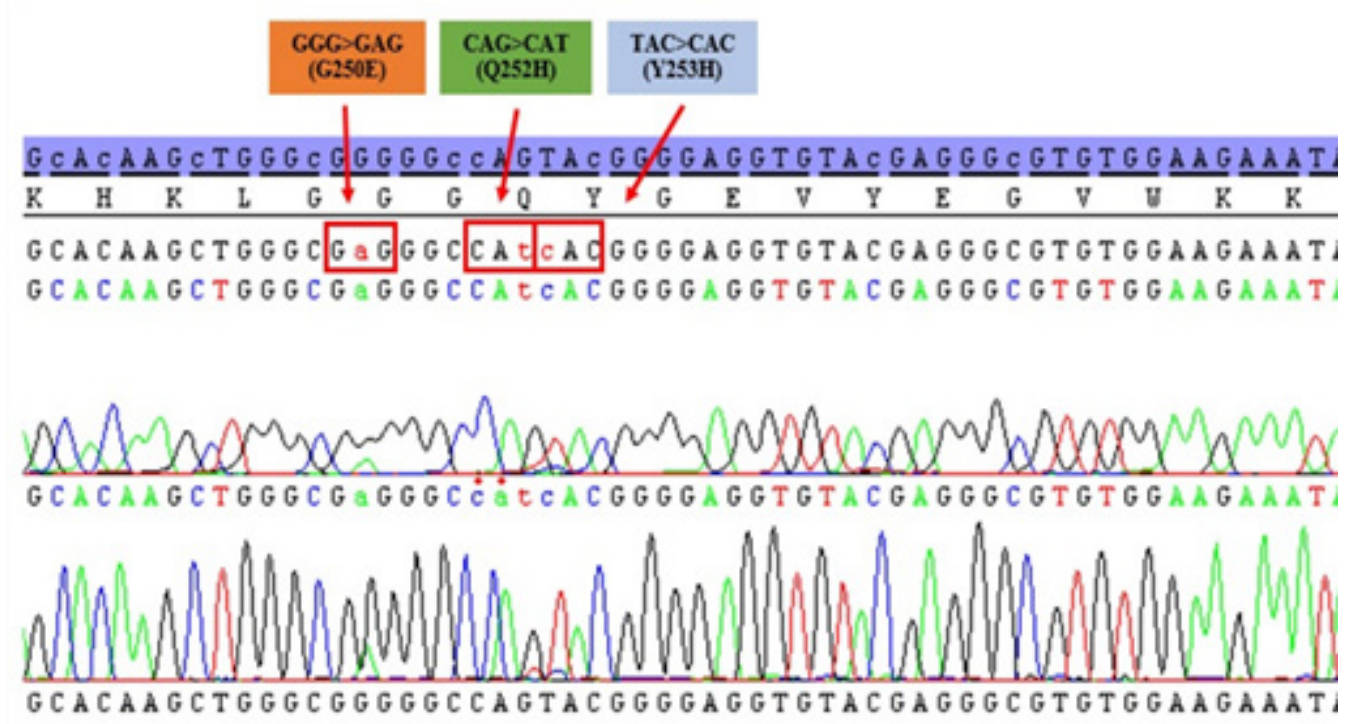

Figure 2. Electropherogram of $B C R-A B L 1$ Tyrosine Kinase Domain Sequencing Profile from Patient with G250E/ Q252H/Y253H Compound/Polyclonal Mutation 


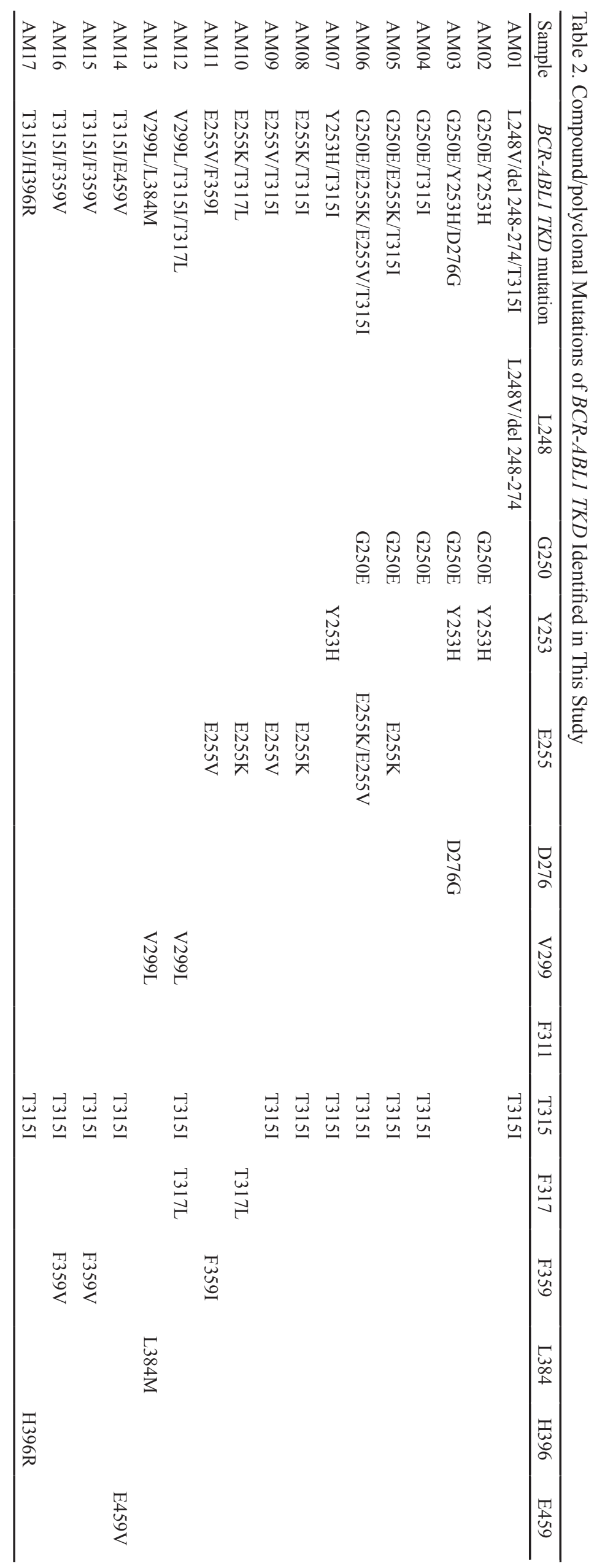



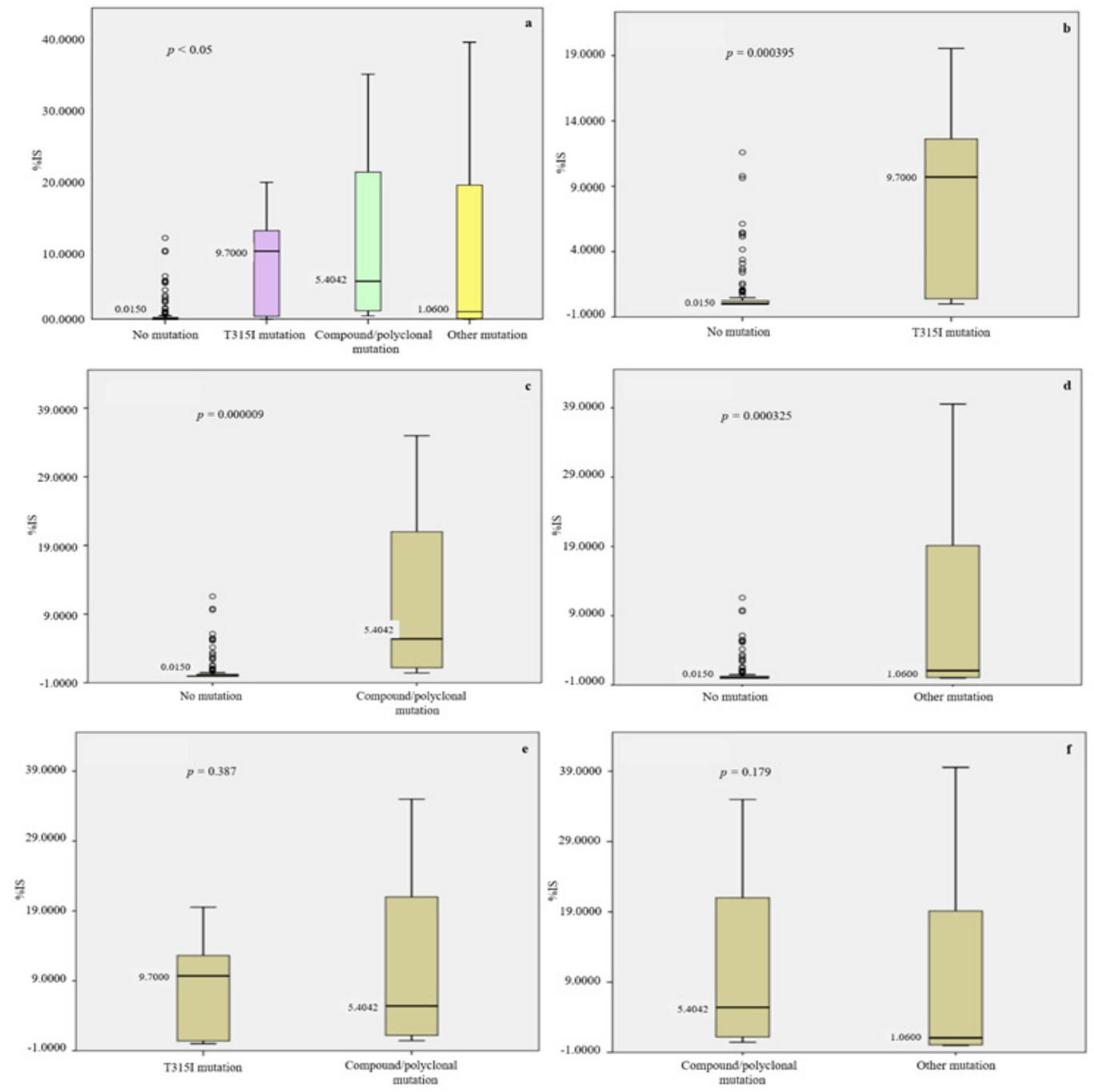

Figure 3. Statistical Analysis of $B C R-A B L 1$ TKD Mutation Statuses and Molecular Response (median \%IS) to Tyrosine Kinase Inhibitors at 12 Months of Monitoring. The overall statistical different in median \%IS among groups as indicated $B C R-A B L T K D$ mutation statuses (a). Comparison of median \%IS between no $B C R-A B L 1$ TKD mutation and T315I (b). Comparison of median \%IS between no BCR-ABL1 TKD mutation and compound/polyclonal with $T 315 I$ (c). Comparison of median \%IS between no BCR-ABL1 TKD mutation and other BCR-ABL1 TKD mutations (d). Comparison of median \%IS between T315I mutation and compound/polyclonal with T315I (e). Comparison of median \%IS between compound/polyclonal with $T 315 I$ and other $B C R$ - $A B L 1$ TKD mutations (f).

mean IS $=8.1510, \%$ median IS $=9.7000)$, compound mutations with T315I $(\mathrm{n}=9 ; \%$ mean IS $=13.0779, \%$ median IS $=5.404)$, and other TKD mutations ( $\mathrm{n}=14 ; \%$ mean IS $=8.1416, \%$ median IS $=1.060)$, respectively. Additionally, an increase of \% IS to upper than $10(>-1$ $\log$ ) was observed in nearly half of patients (44.44\%) with both $T 315 I$ single mutation and compound/polyclonal T315I mutations at 12 months of monitoring. Moreover, statistical analysis indicated that significant different in molecular response was observed in patients without $B C R$ $A B L 1$ TKD mutation compared with patients who carried $B C R-A B L 1$ TKD mutations with T315I ( $\mathrm{p}=0.000395)$,

Table 3. Statistical Analysis by Using Mean and Median \%IS from RQ-PCR Data of Different Group of Patients as Indicated $B C R-A B L 1$ TKD Mutation Sstatuses at 12 Months of Monitoring

\begin{tabular}{lcc}
\hline Compare variable type & $\begin{array}{c}P \text {-value } \\
\text { Compare Mean } \\
\text { (Independent T-test) }\end{array}$ & $\begin{array}{c}\text { Compare Median } \\
\text { (Mann-Whitney) }\end{array}$ \\
\hline No Mutation \& T315I Mutation & 0.015 & $<0.05(0.000395)$ \\
No Mutation \& Compound/polyclonal Mutation with T315I & 0.028 & $<0.05(0.000009)$ \\
No Mutation \& Others Mutation & 0.05 & $<0.05(0.000325)$ \\
T315I Mutation \& Compound/polyclonal Mutation with T315I & 0.364 & 0.387 \\
T315I Mutation \& Others Mutation & 0.998 & 0.643 \\
Compound/polyclonal Mutation with T315I \& Others Mutation & 0.393 & 0.179 \\
\hline
\end{tabular}


Table 4. The Observed Frequency of $B C R-A B L 1$ TKD Mutations in Different Studies

\begin{tabular}{|c|c|c|c|c|}
\hline Study & $\begin{array}{l}\text { Method of the } \\
\text { detection }\end{array}$ & $\begin{array}{l}\text { Overall frequency of } \\
B C R-A B L 1 \text { mutations }(\%)\end{array}$ & $\begin{array}{l}\text { Common hotspot } B C R-A B L 1 \text { TKD } \\
\text { mutations (\%) }\end{array}$ & $\begin{array}{l}\text { CML } \\
\text { phases }\end{array}$ \\
\hline $\begin{array}{l}\text { This study } \\
\text { (Thai study) }\end{array}$ & Direct sequencing & $20(49 / 245)$ & \begin{tabular}{lc}
\multicolumn{3}{l}{ Single mutation $(13)$} \\
Q252H (0.4) & F317L $(1)$ \\
Y253H (0.4) & A350T $(0.4)$ \\
E255K (2) & F359I/V (1) \\
D276G (0.4) & E455K $(0.4)$ \\
F311I (0.4) & E459K $(0.4)$ \\
T315I (7) & F486S (0.4) \\
Compound/polyclonal mutations (7)
\end{tabular} & NA \\
\hline $\begin{array}{l}\text { Elias et al., (2014) } \\
\text { (Malaysian study) } \\
\text { (Elias et al., 2014) }\end{array}$ & $\begin{array}{l}\text { D-HPLC and } \\
\text { Direct sequencing }\end{array}$ & $22(28 / 125)$ & \begin{tabular}{lc}
\multicolumn{2}{l}{ Single mutation } \\
G250E (2) & M351T (2) \\
G251E (1) & E355A/G (2) \\
Y253H (1) & F359C (2) \\
E255K (4) & N368S (1) \\
D276G (1) & L387M (1) \\
V289F (1) & H396R (1) \\
T315I (7) & A397P (1) \\
Compound/polyclonal mutation (NA)
\end{tabular} & CP,AP,BP \\
\hline $\begin{array}{l}\text { Meggyesi et al., } \\
(2012)\end{array}$ & Direct sequencing & $20(15 / 74)$ & Single mutation & $\begin{array}{l}\text { CP,AP,BP, } \\
\text { Ph positive } \\
\text { ALL }\end{array}$ \\
\hline $\begin{array}{l}\text { (Hungarian study) } \\
\text { (Meggyesi et al., } \\
\text { 2012) }\end{array}$ & & & $\begin{array}{ll}\text { M244K/V (8) } & \text { M351T (5) } \\
\text { G250E (1) } & \text { E355G (1) } \\
\text { Y253H (1) } & \text { F359I/V (3) } \\
\text { E255V (4) } & \text { L384M (1) } \\
\text { D276G (1) } & \text { L387M (1) } \\
\text { E279K (1) } & \text { H396R (1) } \\
\text { T315I (5) } & \\
\text { Compound/polyclonal mutation (NA) }\end{array}$ & \\
\hline $\begin{array}{l}\text { Kim et al., (2009) } \\
\text { (Korean syudy) } \\
\text { (Kim et al., 2009) }\end{array}$ & $\begin{array}{l}\text { Direct sequencing } \\
\text { and ASO-PCR }\end{array}$ & $51(70 / 137)$ & 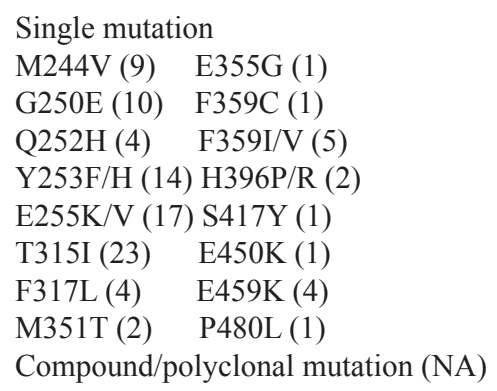 & CP,AP,BP \\
\hline $\begin{array}{l}\text { Branford et al., } \\
\text { (2003) (Australian } \\
\text { study) } \\
\text { (Branford et al., } \\
2003 \text { ) }\end{array}$ & Direct sequencing & $19(27 / 144)$ & \begin{tabular}{ll}
\multicolumn{2}{l}{ Single mutation (14) } \\
M244V (1) & M351T (6) \\
L248V (1) & E355G (2) \\
G250E (1) & F359V (1) \\
Q252H (3) & H396R (1) \\
Y253F (1) & S417Y (1) \\
E255K/V (4) & E459K (1) \\
T315I (1) & F486S (1) \\
F317L (1) & \\
Compound/polyclonal mutations (5)
\end{tabular} & CP,AP \\
\hline
\end{tabular}

compound/polyconal with T315I $(\mathrm{p}=0.000009)$, and other type of $B C R-A B L 1$ mutations ( $\mathrm{p}=0.000325)$, respectively. However, it was not statistical different among patients with T315I, compound/polyconal with T315I, and other $B C R-A B L 1$ mutations observed in this study (table 3 and figure 3 ). Together, we could monitored the molecular response of CML patients after TKI treatment by serially measure the level of BCR-ABL1 fusion transcript and T315I mutation was predominantly identified in most cases with TKI resistant.

\section{Discussion}

During the past two decades, treatment of BCRABL1 positive CML has dramatically improved since the development of imatinib and other tyrosine kinase inhibitors. While the majority of patients remain in prolonged complete molecular response, nearly one third of patient develops an acquired TKD mutations resulting in disease relapse. Several laboratory techniques including conventional cytogenetic, FISH, RT-PCR, quantitative RT-PCR, and digital droplet PCR have been broadly used 
for routine monitoring of Philadelphia positive CML during the treatment. Furthermore, PCR sequencing and recently next generation sequencing technique have been subsequently performed to detect $B C R-A B L 1$ TKD mutational profile in patients who exhibit signs of TKI resistance. Since 2004, we had established a laboratory testing panel for a comprehensive monitoring of CML patients in the country which is continually improved according to the ELN guidelines. Those are including standard karyotyping, commercially dual-color FISH probes, and laboratory developed-test series of RT-PCR for BCR-ABL1, real-time quantitative PCR for BCR-ABL1 mRNA, and direct sequencing technique for $B C R-A B L 1$ TKD mutations. As the quality of each laboratory test is very important, all of those assays have been accredited by the International Organization for Standardization (ISO15189 and ISO15190) as well as participated in the College of American Pathology (CAP) External Quality Assurance (EQA)/Proficiency Testing program.

Perspective of cytogenetic analysis for the diagnosis and monitoring of CML is still limited used and routinely service in Thailand and nearby countries. We use the International System for Human Chromosome Nomenclature (ISCN2016) as a guideline for the description of individual CML karyotype. The main advantages of conventional cytogenetic analysis in CML are including; it is still a gold-standard method for the detection of $\mathrm{Ph}$ chromosome and it could detect several additional chromosomes in both newly diagnosed (baseline) and treated patients. Therefore, the assay is still mandatory for the monitoring of clonal evolution during treatment. However, the technique has some drawbacks such as low resolution, low sensitivity, time consumable, and laborious technique. The molecular cytogenetic, FISH is not frequently used in our laboratory for both diagnosis and monitoring of CML due to the assay is not provide much clinical relevant data compared with conventional cytogenetic analysis. However, we use FISH in special scenarios such as for the monitoring of $\mathrm{Ph}$ positive CML/ AML with rare BCR-ABL1 transcriptional variants.

Since the announcement of European Against Cancer (EAC) procedure for standardization and quality control of real-time quantitative PCR for minimal residual disease detection in leukemia (Gabert et al., 2003), we had followed this valuable protocol for the detection of several fusion transcripts including the BCR-ABL1 major (p210) transcript. This protocol have been proved to be practically used for monitoring CML patient during treatment because it has several benefits such as high specificity and sensitivity, easy to perform, economy, and short turn-around time. More importantly, the result from RQ-PCR is very informative and representing to disease progression/response in individual patient during the treatment. For the international standardization, we could achieve the conversion factor for international scale calculation in 2014 by the support from the Division of Genetics \& Molecular Pathology, Royal Adelaide Hospital, Australia. Here, based-on the ELN 2013 guideline (Baccarani et al., 2013), we serially monitored (every 3 months) BCR-ABL1 mRNA by using RQ-PCR in $245 \mathrm{CML}$ patients after treatment. We observed that the overall response to TKI treatment in patients were gradually decreased at the month 12 after first time point of monitoring (optimal response started from $89 \%$ at month 3 and turned out to be $65 \%$ at month 12 ). Moreover, we found that $22 \%$ of patients were categorized as failure to TKI at month 12 by monitoring with RQ-PCR. Those patients were successively analyzed the BCR-ABL1 TKD profiles using PCR sequencing. While we did not have much clinical therapeutic data of individual patients, we could perform the robust RQ-PCR method for effective monitoring of BCR-ABL1 mRNA in CML during treatment.

Perspective of the detection of $B C R-A B L 1$ TKD mutations in treated patients, we found that $20 \%$ of patients are positive for $B C R-A B L 1$ TKD mutations by mean of using direct sequencing technique. Our data was similar to some of previous publications regarding to the overall frequency of $B C R-A B L 1$ TKD mutations in treated CML. Those were including in the Australian study (19\%) (Branford et al., 2009), Argentinean study (23\%) (Bengio et al., 2011), and Malaysian study (22.4\%) (using dHPLC and direct sequencing) (Elias et al., 2014). However, there were some publications reported that the observed in frequency of $B C R-A B L 1$ TKD mutations are range from $30 \%$ to $65 \%$ (Cortes et al., 2007; Markose et al., 2009; Qin et al., 2011). The overall frequency of $B C R-A B L 1$ TKD mutation in treated CML patients from various studies were summarised in table 4 . The difference in distribution of $B C R-A B L 1$ TKD mutations from various studies could be explained by the variation in number and inclusion criteria of selected case, treatment protocol, phase of disease, and the method of used for the detection of $B C R-A B L 1$ TKD mutations. Similar to previous reports (Branford et al., 2009; Branford et al., 2003; Markose et al., 2009; Nicolini et al., 2006), the most frequents $B C R-A B L 1$ TKD mutations involving in the establishment of TKI resistance by mean of RQ-PCR was the T315I which was accounted for $57 \%$ of all mutated cases. Interestingly, we found that $35 \%$ of all mutated cases persisted compound/polyclonal mutation patterns with dominantly harbour co-mutation of $T 315 I$. Additionally, patients with T315I compound/polyclonal mutations showed higher \%IS and might had phenotypic resistance than those with $T 315 \mathrm{I}$ alone and other TKD mutation patterns at month 12 of monitoring. Although we had no high performance technology such as digital droplet PCR and next generation sequencing to deeply and massively monitor $B C R-A B L 1$ TKD mutation profile, we could perform the effective laboratory developed test (direct sequencing) for the detection of $B C R-A B L 1$ TKD mutations in our tested samples. Based on this observation data, we are going to further investigate the impact of compound/polyclonal mutations in the treatment of $C M L$ in the context of precision medicine in recent future.

In summary, we described the practical laboratory tools for routinely monitoring of CML patients during treatment. Serially measurement of BCR-ABL mRNA using RQ-PCR was able to effectively monitor the dynamic change of residual disease in treated patients. Moreover, we could identify several $B C R-A B L 1$ TKD mutations in patients who failed to TKI therapy. Patients 
with T315I compound/polyclonal mutations had high level of \%IS than those with T315I alone, others TKD mutations, and without TKD mutation. Regarding to ELN 2013 guideline, the integration of laboratory tools including cytogenetic analysis, iFISH, RQ-PCR and direct sequencing is critical for the comprehensive monitoring of $\mathrm{Ph}$ positive $\mathrm{CML}$ during treatment.

\section{Acknowledgements}

We would like to thank Professor Saengsuree Jootar for clinical data and technical guidance. We also would like to thank Professor Prapon Wilairat for paper proofreading. This work was supported by the Thailand Research Fund (TRF MRG 6280227). Roongrudee Singdong is supported by the Royal Golden Jubilee (RGJ) grant for PhD student number PHD/0071/2561.

\section{References}

An X, Tiwari AK, Sun Y, et al (2010). BCR-ABL tyrosine kinase inhibitors in the treatment of Philadelphia chromosome positive chronic myeloid leukemia: a review. Leuk Res, 34, 1255-68.

Baccarani M, Cortes J, Pane F, et al (2009). Chronic myeloid leukemia: an update of concepts and management recommendations of European LeukemiaNet. J Clin Oncol, 27, 6041-51.

Baccarani M, Deininger MW, Rosti G, et al (2013). European LeukemiaNet recommendations for the management of chronic myeloid leukemia: 2013. Blood, 122, 872-84.

Baccarani M, Saglio G, Goldman J, et al (2006). Evolving concepts in the management of chronic myeloid leukemia: recommendations from an expert panel on behalf of the European LeukemiaNet. Blood, 108, 1809-20.

Bengio RM, Riva ME, Moiraghi B, et al (2011). Clinical outcome of chronic myeloid leukemia imatinib-resistant patients: do BCR-ABL kinase domain mutations affect patient survival? First multicenter Argentinean study. Leuk Lymphoma, 52, 1720-6.

Bixby D, Talpaz M (2009). Mechanisms of resistance to tyrosine kinase inhibitors in chronic myeloid leukemia and recent therapeutic strategies to overcome resistance. Hematol Am Soc Hematol Educ Program, 2009, 461-76.

Branford S, Hughes T (2006). Detection of BCR-ABL mutations and resistance to imatinib mesylate. Methods $\mathrm{Mol} \mathrm{Med}$, 125, 93-106.

Branford S, Melo JV, Hughes TP (2009). Selecting optimal second-line tyrosine kinase inhibitor therapy for chronic myeloid leukemia patients after imatinib failure: does the BCR-ABL mutation status really matter?. Blood, 114, 5426-35.

Branford S, Rudzki Z, Walsh S, et al (2003). Detection of BCR$\mathrm{ABL}$ mutations in patients with CML treated with imatinib is virtually always accompanied by clinical resistance, and mutations in the ATP phosphate-binding loop (P-loop) are associated with a poor prognosis. Blood, 102, 276-83.

Breccia M, Molica M, Colafigli G, Massaro F, Alimena G (2016). Early molecular response in chronic myeloid leukemia and halving time: Latest evidences. Leuk Res, 48, 20-5.

Buchdunger E, Matter A, Druker BJ (2001). Bcr-Abl inhibition as a modality of CML therapeutics. Biochim Biophys Acta, 1551, M11-8.

Calabretta B, Perrotti D (2004). The biology of CML blast crisis. Blood, 103, 4010-22.
Chhikara S, Sazawal S, Seth T, et et (2017). Molecular response to imatinib and its correlation with mRNA expression levels of imatinib influx transporter (OCT1) in Indian chronic myeloid leukemia patients. Asian Pac J Cancer Prev, 18, 2043-8.

Cooper S, Giles FJ, Savona MR (2009). Overcoming resistance in chronic myelogenous leukemia. Leuk Lymphoma, 50, 1785-93.

Cortes J, Jabbour E, Kantarjian H, et al (2007). Dynamics of BCR-ABL kinase domain mutations in chronic myeloid leukemia after sequential treatment with multiple tyrosine kinase inhibitors. Blood, 110, 4005-11.

Druker BJ, Lydon NB (2000). Lessons learned from the development of an abl tyrosine kinase inhibitor for chronic myelogenous leukemia. J Clin Invest, 105, 3-7.

Druker BJ, Talpaz M, Resta DJ, et al (2001). Efficacy and safety of a specific inhibitor of the BCR-ABL tyrosine kinase in chronic myeloid leukemia. N Engl J Med, 344, 1031-7.

Elias MH, Baba AA, Azlan H, et al (2014). BCR-ABL kinase domain mutations, including 2 novel mutations in imatinib resistant Malaysian chronic myeloid leukemia patients-Frequency and clinical outcome. Leuk Res, 38, 454-9.

Elias MH, Baba AA, Husin A, et al (2013). HOXA4 gene promoter hypermethylation as an epigenetic mechanism mediating resistance to imatinib mesylate in chronic myeloid leukemia patients. Biomed Res Int, 2013, 129715.

Gabert J, Beillard E, van der Velden VH, et al (2003). Standardization and quality control studies of 'real-time' quantitative reverse transcriptase polymerase chain reaction of fusion gene transcripts for residual disease detection in leukemia - a Europe Against Cancer program. Leukemia, 17, 2318-57.

Goldman JM, Melo JV (2003). Chronic myeloid leukemia-advances in biology and new approaches to treatment. $N$ Engl J Med, 349, 1451-64.

Hehlmann R, Hochhaus A, Baccarani M, European L (2007). Chronic myeloid leukaemia. Lancet, 370, 342-50.

Jabbour E, Cortes JE, Kantarjian HM (2008). Molecular monitoring in chronic myeloid leukemia: response to tyrosine kinase inhibitors and prognostic implications. Cancer, 112, 2112-8.

Jabbour E, Kantarjian H (2014). Chronic myeloid leukemia: 2014 update on diagnosis, monitoring, and management. Am J Hematol, 89, 547-56.

Kaleem B, Shahab S, Ahmed N, Shamsi TS (2015). Chronic myeloid leukemia--prognostic value of mutations. Asian Pac J Cancer Prev, 16, 7415-23.

Kim SH, Kim D, Kim DW, et al (2009). Analysis of Bcr-Abl kinase domain mutations in Korean chronic myeloid leukaemia patients: poor clinical outcome of P-loop and T315I mutation is disease phase dependent. Hematol Oncol, 27, 190-7.

Lim YM, Eng WL, Chan HK (2017). Understanding and challenges in taking tyrosine kinase inhibitors among Malaysian chronic myeloid leukemia patients: A Qualitative Study. Asian Pac J Cancer Prev, 18, 1925-30.

Markose P, Chendamarai E, Balasubramanian P, et al (2009). Spectrum of BCR-ABL kinase domain mutations in patients with chronic myeloid leukemia from India with suspected resistance to imatinib-mutations are rare and have different distributions. Leuk Lymphoma, 50, 2092-5.

Meggyesi N, Kozma A, Halm G, et al (2012). Additional chromosome abnormalities, BCR-ABL tyrosine kinase domain mutations and clinical outcome in Hungarian tyrosine kinase inhibitor-resistant chronic myelogenous leukemia patients. Acta Haematol, 127, 34-42. 
Nicolini FE, Corm S, Le QH, et al (2006). Mutation status and clinical outcome of 89 imatinib mesylate-resistant chronic myelogenous leukemia patients: a retrospective analysis from the French intergroup of CML (Fi(phi)-LMC GROUP). Leukemia, 20, 1061-6.

Patel AB, O'Hare T, Deininger MW (2017). Mechanisms of resistance to $\mathrm{ABL}$ kinase inhibition in chronic myeloid leukemia and the development of next generation ABL kinase inhibitors. Hematol Oncol Clin North Am, 31, 589-612.

Qin Y, Chen S, Jiang B, et al (2011). Characteristics of BCR-ABL kinase domain point mutations in Chinese imatinib-resistant chronic myeloid leukemia patients. Ann Hematol, 90, 47-52.

Savage DG, Antman KH (2002). Imatinib mesylate--a new oral targeted therapy. $N$ Engl J Med, 346, 683-93.

Shah K, Parikh S, Rawal R (2016). Tyrosine kinase inhibitors in $\mathrm{Ph}+$ chronic myeloid leukemia therapy: a Review. Asian Pac J Cancer Prev, 17, 3025-33.

Talpaz M, Silver RT, Druker BJ, et al (2002). Imatinib induces durable hematologic and cytogenetic responses in patients with accelerated phase chronic myeloid leukemia: results of a phase 2 study. Blood, 99, 1928-37.

Tantiworawit A, Kongjarern S, Rattarittamrong E, et al (2016). Diagnosis and monitoring of chronic myeloid leukemia: Chiang Mai University Experience. Asian Pac J Cancer Prev, 17, 2159-64.

Tauchi T, Ohyashiki K (2004). Molecular mechanisms of resistance of leukemia to imatinib mesylate. Leuk Res, 28, 39-45.

Thompson PA, Kantarjian HM, Cortes JE (2015). Diagnosis and Treatment of Chronic Myeloid Leukemia in 2015. Mayo Clin Proc, 90, 1440-54.

Vacarean-Trandafir IC, Ivanov IC, Dragos LM, et al (2019). ABL1 tyrosine kinase domain mutations in chronic myeloid leukemia treatment resistance. Mol Biol Rep, 46, 3747-54.

Yang K, Fu LW (2015). Mechanisms of resistance to BCR-ABL TKIs and the therapeutic strategies: A review. Crit Rev Oncol Hematol, 93, 277-92.

\section{(c) (i) (8)}

This work is licensed under a Creative Commons AttributionNon Commercial 4.0 International License. 